\title{
Monte Carlo simulation and measurement of radiation leakage from applicators used in external electron radiotherapy
}

\author{
Tomohiro Shimozato $^{a, *}$, Kuniyasu Okudaira ${ }^{b}$, Hiraku Fuse ${ }^{c}$, \\ Katsuyoshi Tabushi ${ }^{\mathrm{d}}$
}

\author{
a Department of Radiation Oncology, Iwata City Hospital, 512-3 Ohkubo, Iwata, Shizuoka 4388550, \\ Japan \\ b Department of Radiotherapy, Nagoya University Hospital, 65 Tsuruma-chou, Shouwa-ku, \\ Nagoya, Aichi 4668560, Japan \\ ' Proton Medical Research Center, University of Tsukuba, 2-2-1 Amakudo, Tsukuba, \\ Ibaraki 3058576, Japan \\ 'Department of Radiological Technology, Nagoya University School of Health Science, \\ 1-1-20 Daikoh-minami,Higashi-ku, Nagoya, Aichi 4618673, Japan
}

Received 23 January 2012; received in revised form 27 May 2012; accepted 9 June 2012 Available online 7 July 2012

\section{KEYWORDS \\ External electron \\ radiotherapy; \\ Peripheral dose; \\ Monte Carlo \\ simulation}

\begin{abstract}
External electron radiotherapy is performed using a cone or applicator to collimate the beam. However, because of a trade-off between collimation and scattering/bremsstrahlung X-ray production, applicators generate a small amount of secondary radiation (leakage). We investigate the peripheral dose outside the radiation field of a Varian-type applicator. The dose and fluence outside the radiation field were analyzed in a detailed Monte Carlo simulation. The differences between the calculation results and data measured in a water phantom in an ionization chamber were less than $\pm 1 \%$ in regions more than $3 \mathrm{~mm}$ below the surface of the phantom and at the depth of dose maximum. The calculated fluence was analyzed inside and outside the radiation field on a plane just above the water phantom surface. Changing the electron energy affected the off-axis fluence distribution outside the radiation field; however, the size of the applicator had little effect on this distribution. For each energy, the distributions outside the radiation field were similar to the dose distribution at shallow depths in the water phantom. The effect of secondary electrons generation by photon transmission through the alloy making up the lowest scraper was largest in the region from the field edge to directly below the cutout and at higher beam energies. The results of the Monte Carlo simulation
\end{abstract}

\footnotetext{
${ }^{*}$ Corresponding author.

E-mail addresses: shimo-p@umin.ac.jp, shimo-p@umin.net (T. Shimozato).
} 
confirm that the peripheral dose outside the field is significantly affected by radiation scattered or transmitted from the applicator, and the effect increases with the electron energy. (c) 2012 Associazione Italiana di Fisica Medica. Published by Elsevier Ltd. All rights reserved.

\section{Introduction}

In external electron radiotherapy, irradiation is generally performed using an applicator to collimate the beam and reduce radiation leakage to tissue outside the field. However, special irradiation methods such as total skin electron irradiation [1] are exceptions to this practice. Applicators are designed to absorb most incident electrons; however, X-ray photons are transmitted and generate secondary electrons, which in turn increase the absorbed dose. Many papers [2-15] have been published on radiation leakage outside the radiation field. Measurements using films [2-7] and ionization chambers [2,3,5,7-12] have been performed outside the field collimated by electron applicators. Previous papers [8,10-14] analyzed the scattered radiation inside the radiation field by analyzing dose distributions in a water phantom by using Monte Carlo (MC) simulations. For example, Ebert et al. [15] analyzed the scattered and transmitted radiation outside the radiation field of the Siemens KD2 linear accelerator using the MC method. In 1990, Perec and Kubo [4] reduced the peripheral

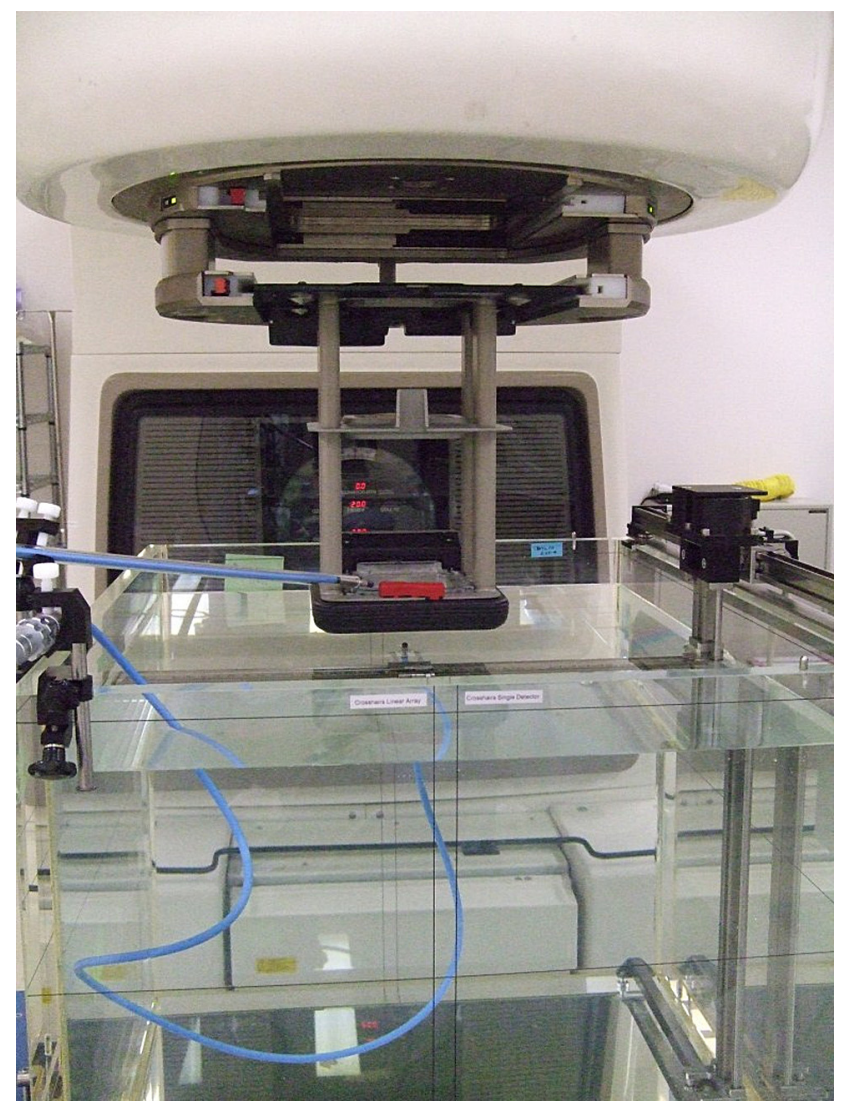

Figure 1 Experimental set up for depth profile and off-axis profile measurement using the ionization chamber and the 3D water phantom. The parallel plane ionization chamber was used to measure the depth dose profile, and the thimble ionization chamber was used to measure the off-axis profile. dose by improving the applicator design. However, the applicator developed by Varian has many gaps and open sidewalls, so it is possible that radiation generated by this type of applicator cannot be reduced sufficiently. Chow and Grigorov [6] later confirmed the presence of peripheral doses outside of Variantype applicators by measurements using films. They reported a peripheral dose of about $1.5 \%$ for a $4-\mathrm{MeV}$ electron beam.

In this investigation, we analyze the contributions of radiation scattered and transmitted by the applicator to the dose outside the radiation field of a Varian-type applicator. We compare the results of an MC simulation with those of measurements in a water phantom in an ionization chamber.

\section{Material and methods}

\section{Measurements}

To acquire the percentage depth ionization (PDI) and dose profiles in a water phantom, the medical linear accelerator Clinac 2100CD (Varian Medical Systems, Palo Alto, CA, USA) and the 3D water phantom mp3 (PTW Freiburg, Freiburg, Germany) were set up as shown in Fig. 1. The Clinac 2100CD allows a choice of five electron energies $(4,6,9,12$, and $16 \mathrm{MeV})$ and a set of five applicators $(6 \times 6,10 \times 10,15 \times 15$, $20 \times 20$, and $25 \times 25 \mathrm{~cm}^{2}$ ). Table 1 [16] lists the secondary jaw settings for each applicator and each energy as defined by the manufacturer. The multi-leaf collimator is normally retracted and is not supposed to affect the beam in electron mode. Varian-type applicators have open sidewalls and three scrapers made of a zinc alloy. The lowest scraper, called the standard insert, is made of zinc alloy and another alloy (58\% $\mathrm{Bi}, 42 \% \mathrm{Sn})$; it is provided by the manufacturer and is used during measurement or treatment to form the radiation field [16]. The side of the collimator nearest the patient is $95 \mathrm{~cm}$ from the source. The design drawing of the electron applicators does not describe the width of each scraper in detail, although the thickness of each scraper is given. We measured the data that did not appear in the design drawing by using a micrometer caliper. The source-to-surface distance (SSD) was $100 \mathrm{~cm}$.

Table 1 Secondary jaw sizes (in centimeters) with electron beam applicator size and nominal electron energy. All field sizes are defined at the isocenter distance $(100 \mathrm{~cm})$ and $X \times Y$ as defined by the manufacturer [16].

\begin{tabular}{llllll}
\hline $\begin{array}{l}\text { Energy } \\
(\mathrm{MeV})\end{array}$ & \multicolumn{4}{l}{ Applicator field size at isocenter $(\mathrm{cm} \times \mathrm{cm})$} \\
\cline { 2 - 6 } & $6 \times 6$ & $10 \times 10$ & $15 \times 15$ & $20 \times 20$ & $25 \times 25$ \\
\hline 4 & $20 \times 20$ & $20 \times 20$ & $20 \times 20$ & $25 \times 25$ & $30 \times 30$ \\
6 & $20 \times 20$ & $20 \times 20$ & $20 \times 20$ & $25 \times 25$ & $30 \times 30$ \\
9 & $20 \times 20$ & $20 \times 20$ & $20 \times 20$ & $25 \times 25$ & $30 \times 30$ \\
12 & $11 \times 11$ & $14 \times 14$ & $17 \times 17$ & $25 \times 25$ & $30 \times 30$ \\
16 & $11 \times 11$ & $14 \times 14$ & $17 \times 17$ & $23 \times 23$ & $28 \times 28$ \\
\hline
\end{tabular}




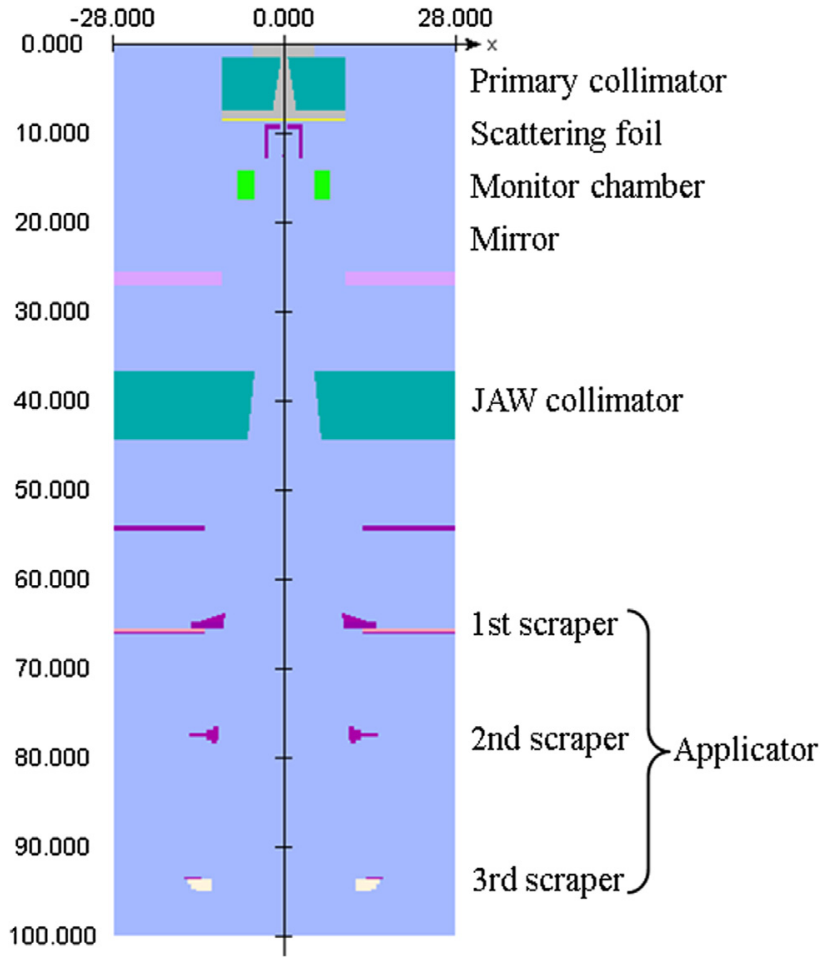

Figure 2 Schematic geometry of the linear accelerator's gantry head for MC simulation using BEAMnrc code. All dimensions are in centimeters.

A TM34045 (PTW Freiburg) parallel plane ionization chamber was used to measure the PDI in the water phantom. The PDI was converted to the percentage depth dose (PDD) according to AAPM TG51 [17] using the dose analysis software MEPHYSTO Ver. 7.42 (PTW Freiburg). Next, the off-axis ratios (OARs) on the cross-line (transverse direction) dose profiles were measured at a depth of $5 \mathrm{~mm}\left(R_{s}\right)$, the depth of dose maximum $\left(R_{100}\right)$, the depth of a $90 \%$ dose $\left(R_{90}\right)$, the depth of a $50 \%$ dose $\left(R_{50}\right)$, and the practical range $\left(R_{p}\right)$ as defined by AAPM TG25 [18] using a TM31010 (PTW Freiburg) thimble ionization chamber with an effective volume of $0.125 \mathrm{~cm}^{3}$. The doses measured at off-axis points of interest were normalized to the dose at the central beam axis in a water phantom for the given electron energy.

\section{Monte Carlo simulation}

\section{Establishment of linear accelerator gantry head configuration}

The MC simulation was performed using EGSnrc [19]. The treatment head was modeled using BEAMnrc [20]. The

Table 2 Parameters of incident electrons in $M C$ simulation.

\begin{tabular}{llll}
\hline $\begin{array}{l}\text { Energy } \\
(\mathrm{MeV})\end{array}$ & $\begin{array}{l}\text { Spot size } \\
(\mathrm{cm})\end{array}$ & $\begin{array}{l}\text { Mean energy } \\
(\mathrm{MeV})\end{array}$ & $\begin{array}{l}\text { eFWHM } \\
(\%)\end{array}$ \\
\hline 4 & 0.20 & 4.27 & 3.0 \\
6 & 0.15 & 6.90 & 3.0 \\
9 & 0.17 & 9.90 & 3.0 \\
12 & 0.15 & 13.40 & 3.0 \\
16 & 0.17 & 17.40 & 3.0 \\
\hline
\end{tabular}

Table 3 Differences (calculated minus measured) in percentage depth dose values for $10 \times 10 \mathrm{~cm}^{2}$ applicator at each energy.

\begin{tabular}{llllll}
\hline $\begin{array}{l}\text { Depth } \\
(\mathrm{cm})\end{array}$ & \multicolumn{6}{l}{ Energy $(\mathrm{MeV})$} & & & \\
\cline { 2 - 6 } & 4 & 6 & 9 & 12 & 16 \\
\hline 0.50 & $-0.98 \%$ & $-1.00 \%$ & $-0.98 \%$ & $-0.97 \%$ & $-0.88 \%$ \\
0.75 & $0.00 \%$ & - & - & - & - \\
1.00 & $1.00 \%$ & $-0.84 \%$ & $-0.89 \%$ & $-0.98 \%$ & $-0.93 \%$ \\
1.40 & - & $0.00 \%$ & - & - & - \\
1.50 & $-0.80 \%$ & $0.21 \%$ & $-0.61 \%$ & $-0.47 \%$ & $-0.39 \%$ \\
2.00 & $-0.17 \%$ & $0.76 \%$ & $-0.36 \%$ & $-0.05 \%$ & $-0.97 \%$ \\
2.20 & - & - & $0.00 \%$ & - & - \\
2.50 & $0.00 \%$ & $0.15 \%$ & $0.57 \%$ & $-0.16 \%$ & $-0.73 \%$ \\
2.80 & - & - & - & $0.00 \%$ & - \\
3.00 & $-0.01 \%$ & $-0.69 \%$ & $0.93 \%$ & $-0.04 \%$ & $-0.18 \%$ \\
3.50 & $-0.01 \%$ & $-0.03 \%$ & $0.92 \%$ & $-0.20 \%$ & $0.00 \%$ \\
4.00 & $0.00 \%$ & $-0.01 \%$ & $0.47 \%$ & $0.26 \%$ & $0.15 \%$ \\
4.50 & - & $0.02 \%$ & $-0.15 \%$ & $-0.12 \%$ & $0.27 \%$ \\
5.00 & - & - & $-0.05 \%$ & $-0.36 \%$ & $0.42 \%$ \\
5.50 & - & - & $0.03 \%$ & $-0.74 \%$ & $0.12 \%$ \\
6.00 & - & - & - & $-0.59 \%$ & $0.01 \%$ \\
6.50 & - & - & - & - & $-0.21 \%$ \\
\hline
\end{tabular}

treatment head configuration consisted of a primary collimator, exit window, primary scattering foil, secondary scattering foil, monitor chamber, mirror, upper and lower jaws, Mylar screen, and applicator, as shown in Fig. 2. The manufacturer provided detailed information about the geometry, material composition, and dimensions of each component under a confidential agreement. The boundary crossing algorithm was set to EXACT, and spin effects were turned on. The electron step algorithm was PRESTA-II, and

Table 4 Differences (calculated minus measured) in offaxis ratios for $10 \times 10 \mathrm{~cm}^{2}$ applicator at 5-mm depth $\left(R_{s}\right)$ for each energy.

\begin{tabular}{lrlrrr}
\hline $\begin{array}{l}\text { Distance } \\
\text { from }\end{array}$ & \multicolumn{5}{l}{ Energy (MeV) } \\
\cline { 2 - 6 } $\begin{array}{l}\text { central } \\
\text { axis (cm) }\end{array}$ & \multicolumn{1}{l}{6} & \multicolumn{1}{l}{12} & \multicolumn{1}{l}{16} \\
\hline 0.00 & $0.00 \%$ & $0.00 \%$ & $0.00 \%$ & $0.00 \%$ & $0.00 \%$ \\
1.00 & $0.50 \%$ & $0.89 \%$ & $0.75 \%$ & $-0.41 \%$ & $1.00 \%$ \\
2.00 & $0.39 \%$ & $-0.75 \%$ & $-0.26 \%$ & $-0.75 \%$ & $0.98 \%$ \\
3.00 & $-0.14 \%$ & $-0.32 \%$ & $1.00 \%$ & $0.13 \%$ & $0.64 \%$ \\
4.00 & $1.31 \%$ & $-1.19 \%$ & $0.59 \%$ & $1.43 \%$ & $1.78 \%$ \\
5.00 & $-1.71 \%$ & $-3.33 \%$ & $-3.89 \%$ & $-3.90 \%$ & $-0.51 \%$ \\
6.00 & $-1.86 \%$ & $-0.73 \%$ & $-0.45 \%$ & $-0.79 \%$ & $-1.22 \%$ \\
7.00 & $-0.08 \%$ & $-0.06 \%$ & $-0.06 \%$ & $-0.19 \%$ & $-0.34 \%$ \\
8.00 & $0.02 \%$ & $0.00 \%$ & $-0.14 \%$ & $-0.21 \%$ & $-0.35 \%$ \\
9.00 & $-0.01 \%$ & $0.01 \%$ & $0.00 \%$ & $-0.12 \%$ & $-0.15 \%$ \\
10.00 & $0.00 \%$ & $0.05 \%$ & $0.06 \%$ & $0.02 \%$ & $-0.10 \%$ \\
11.00 & $-0.03 \%$ & $0.04 \%$ & $0.04 \%$ & $0.05 \%$ & $0.03 \%$ \\
12.00 & $0.01 \%$ & $0.08 \%$ & $0.10 \%$ & $0.10 \%$ & $0.09 \%$ \\
13.00 & $0.12 \%$ & $0.18 \%$ & $0.19 \%$ & $0.20 \%$ & $0.15 \%$ \\
14.00 & $0.23 \%$ & $0.15 \%$ & $0.15 \%$ & $0.16 \%$ & $0.17 \%$ \\
15.00 & $0.22 \%$ & $0.14 \%$ & $0.09 \%$ & $0.11 \%$ & $0.18 \%$ \\
16.00 & $0.11 \%$ & $0.19 \%$ & $0.17 \%$ & $0.16 \%$ & $0.12 \%$ \\
\hline & & & & &
\end{tabular}



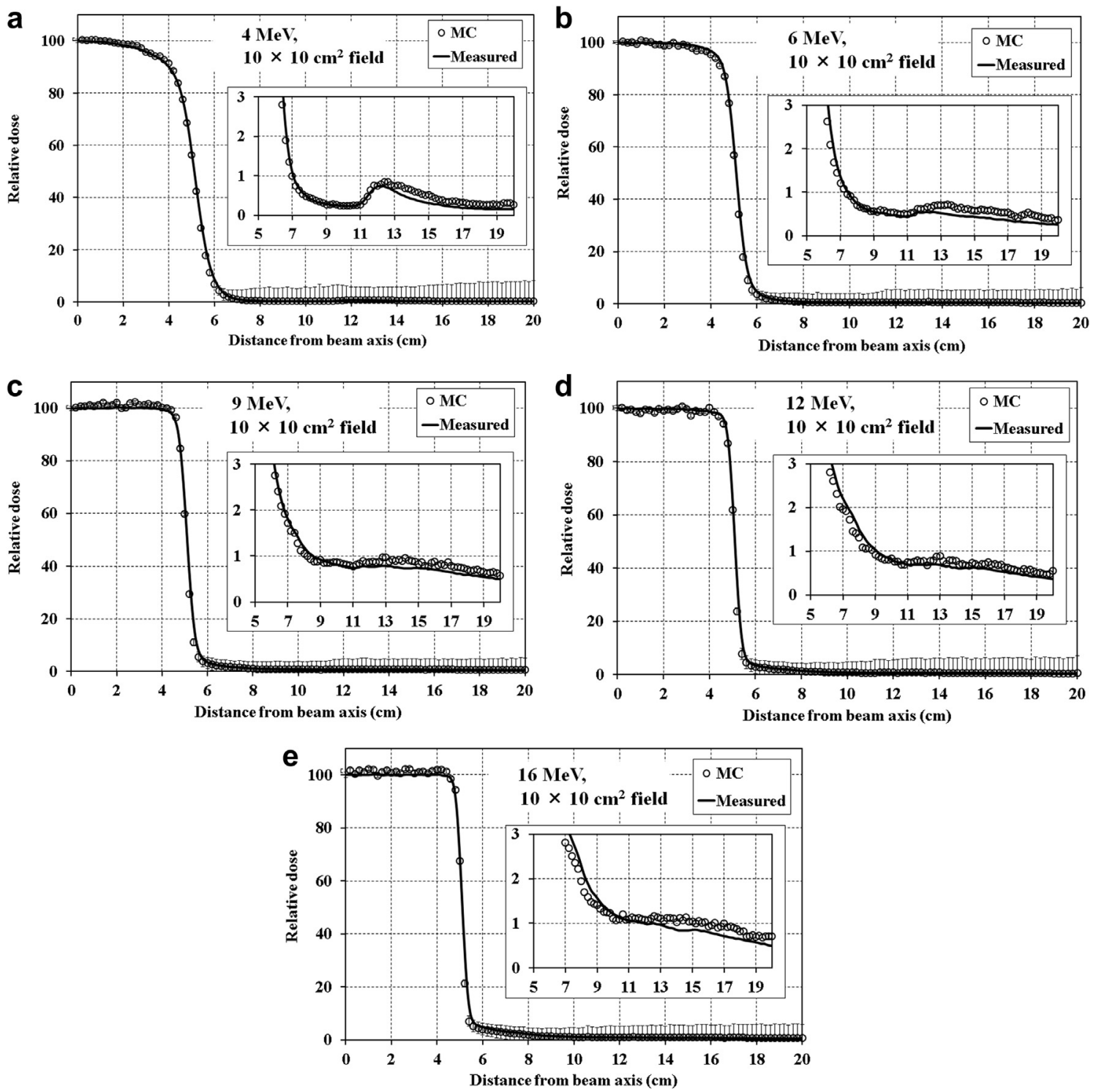

Figure 3 Dose distributions in the cross-line (transverse) direction as measured by the ionization chamber (solid lines) and calculated using the MC simulation (circles) at 5-mm depth $\left(R_{s}\right)$ in the water phantom at beam energies of (a) $4 \mathrm{MeV}$, (b) $6 \mathrm{MeV}$, (c) $9 \mathrm{MeV}$, (d) $12 \mathrm{MeV}$, and (e) $16 \mathrm{MeV}$ for the $10 \times 10 \mathrm{~cm}^{2}$ applicator. Each data point was normalized by the depth of dose maximum for each energy. Insets show magnified graphs outside the field edge. Error bars indicate one standard deviation results calculated using the MC simulation.

the maximum fractional energy loss per step (ESTEPE) was set to 0.25. In BEAMnrc, transport parameters of $\mathrm{AP}=\mathrm{PCUT}=0.01 \mathrm{MeV}$ and $\mathrm{AE}=\mathrm{ECUT}=0.521 \mathrm{MeV}$ were selected. Phase space data were scored at $100 \mathrm{~cm}$ SSD. The secondary jaws for each applicator were set to the positions specified by the manufacturer (Table 1). The source was simulated with a Gaussian energy distribution using ISOURC $=19$ in BEAMnrc, which represents a parallel circular beam with a Gaussian radial distribution [21].

Phase space data were used as input to compute the dose distribution in a heterogeneous water phantom using the EGSnrc/DOSXYZnrc code [22]. In DOSXYZnrc, transport parameters of $\mathrm{AP}=\mathrm{PCUT}=0.01 \mathrm{MeV}$ and $\mathrm{AE}=\mathrm{ECUT}=0.521 \mathrm{MeV}$ were selected. The mean energies, energy spread, and spatial spread of the incident electron beam were adjusted iteratively until the measured and simulated PDD and OAR values agreed to within $\pm 1 \%$.

Table 2 lists the parameters of the incident electrons for each energy: the spatial spread (spot size, shown as full width at half maximum [FWHM]), mean energy, and eFWHM (FWHM of energy spread). The number of particle histories was determined such that the statistical uncertainty would be within $\pm 1 \%$ for each PDD in the region from $R_{100}$ to $R_{p}$ and for the OARs inside the radiation field at $R_{100}$. The number of primary electrons ranged from 200 million to 400 million for the BEAMnrc simulation, depending on the electron energy. For the DOSXYZnrc simulation, the number of histories was 5000 million. The material library PEGS4 (Preprocessor for EGS4) data file was used, and materials that did not appear by default in PEGS4 were added and 

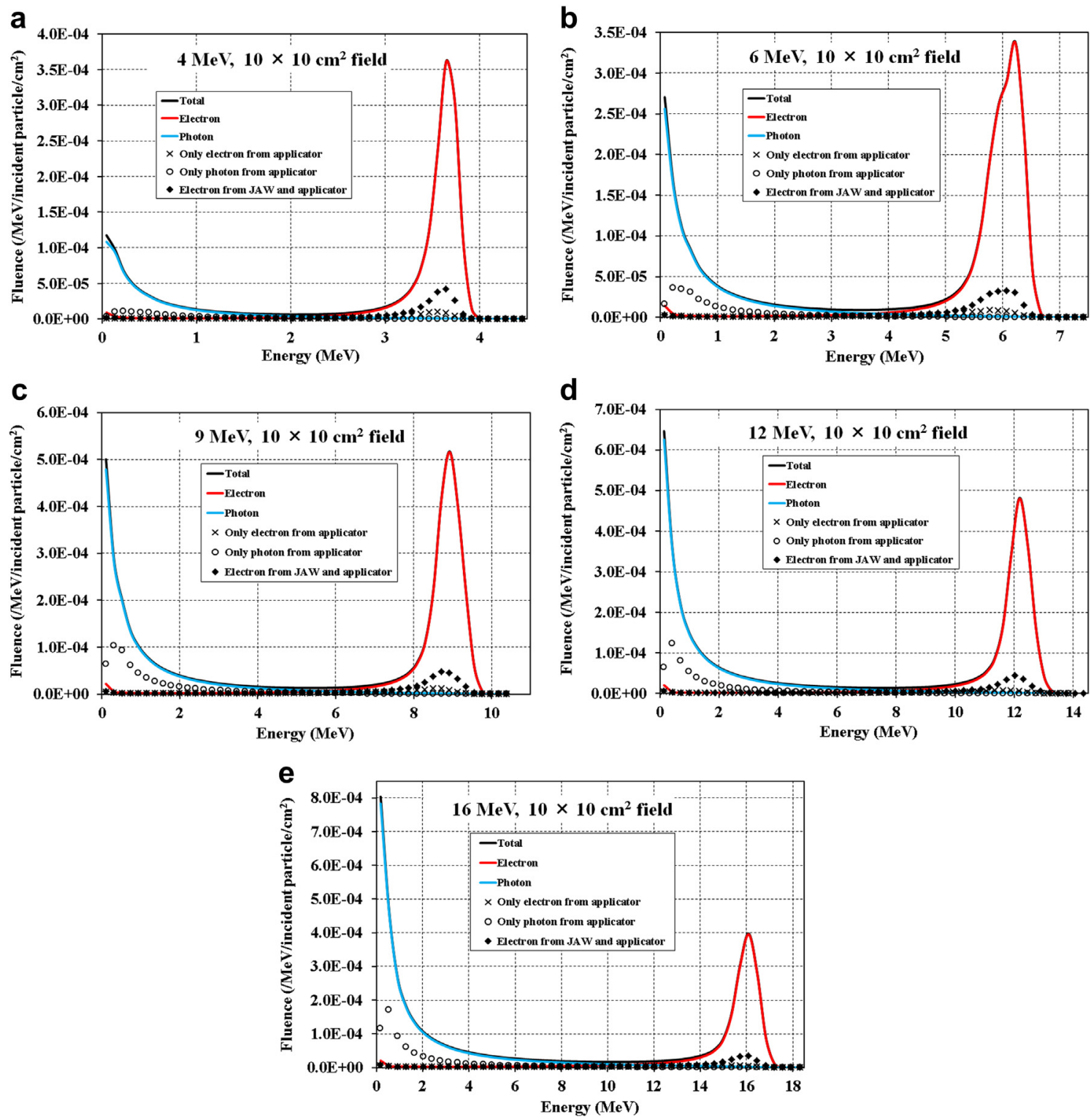

Figure 4 Fluence distribution inside the radiation field for (a) $4 \mathrm{MeV}$, (b) $6 \mathrm{MeV}$, (c) $9 \mathrm{MeV}$, (d) $12 \mathrm{MeV}$, and (e) $16 \mathrm{MeV}$ electron beams for the $10 \times 10 \mathrm{~cm}^{2}$ applicator.

calculated by EGSnrcMP (EGSnrc multi-platform) [23]. The computation times ranged from 216 to $729 \mathrm{~h}$ for BEAMnrc simulation and from 92 to $221 \mathrm{~h}$ for the DOSXYZnrc simulation, depending on the incident electron energy. The simulation was performed in Microsoft Windows XP SP2 with the g77 compiler installed on an Intel Core ${ }^{\mathrm{TM} 2}$ Duo E6850 3.0 GHz processor with $2.0 \mathrm{~GB}$ of RAM.

The calculation grid size was $0.1 \times 0.1 \times 0.1 \mathrm{~cm}^{3}$. STATDOSE [24] was used to extract the PDDs and OARs from the DOSXYZnrc water phantom dose distributions. The size of the geometrical field was defined as $100 \mathrm{~cm} \mathrm{SSD}$. The size of the radiation field was defined as the FWHM when the OAR value was normalized on the central beam axis at $100 \mathrm{~cm}$ SSD. The difference between the geometrical and radiation fields was maintained at less than $2 \mathrm{~mm}$ or $\pm 1 \%$, in accordance with AAPM TG40 [25].

\section{Analysis of scattered and transmitted radiation from} applicator

BEAMDP [26] was used to analyze the phase space data acquired in BEAMnrc. The 2D distributions were analyzed, and the spectral fluence distributions were generated for the selected component using the LATCH option to identify the type and position of scattered radiation. Consequently, the dose distribution and fluence generated by scattered radiation from various components could be analyzed.

The spectral fluence inside and outside the radiation field for each applicator was obtained using BEAMDP to investigate the differences in the fluence distribution due to variations in the applicator size or electron energy. The purpose was to investigate the contributions of transmitted and scattered radiation to the dose distribution in the water phantom. 
a

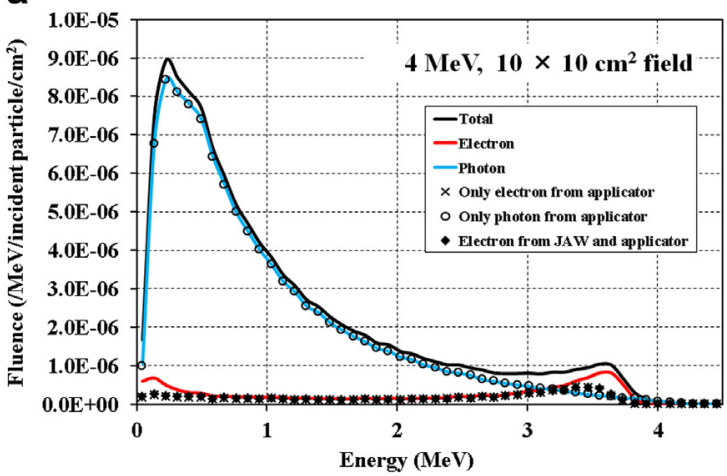

C

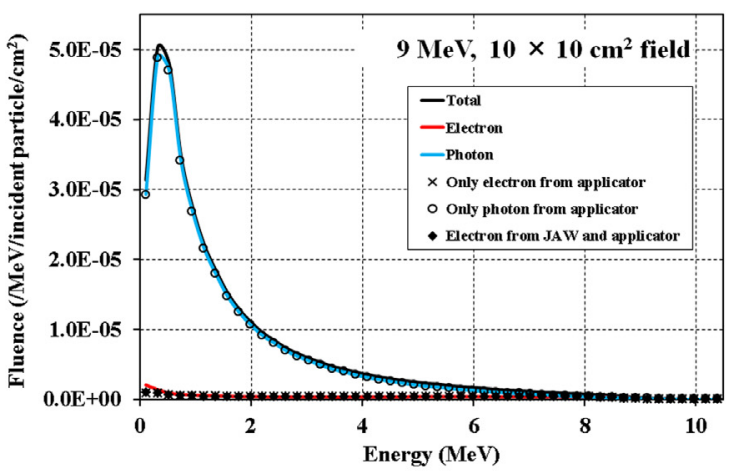

b

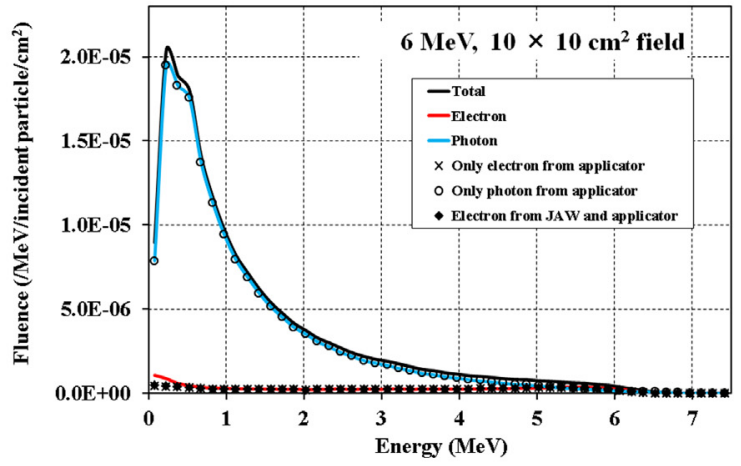

d

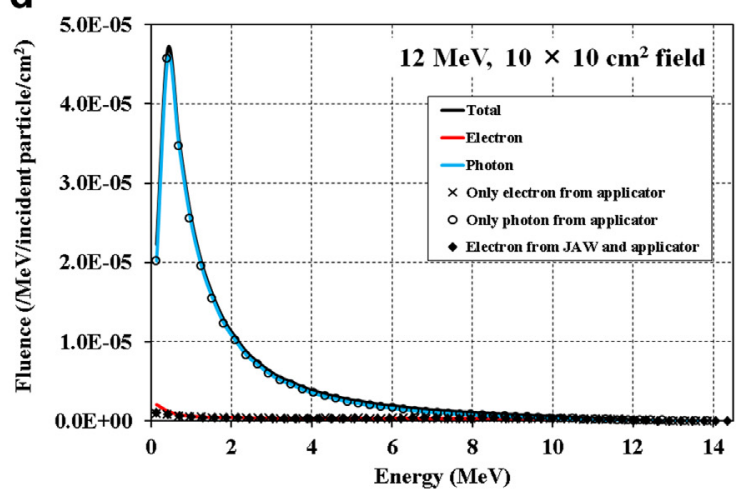

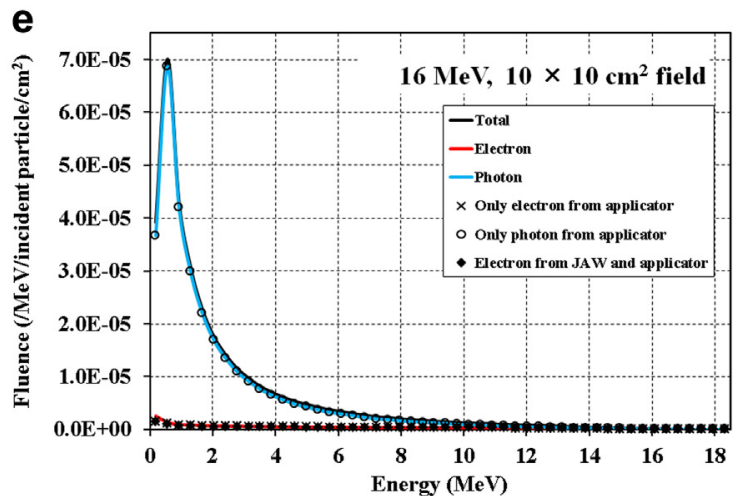

Figure 5 Fluence distribution outside the radiation field for (a) $4 \mathrm{MeV}$, (b) $6 \mathrm{MeV}$, (c) $9 \mathrm{MeV}$, (d) $12 \mathrm{MeV}$, and (e) $16 \mathrm{MeV}$ electron beams for the $10 \times 10 \mathrm{~cm}^{2}$ applicator.

\section{Results and discussion}

\section{Comparison of calculated and measured data}

The differences between the calculated and measured PDD values were less than $\pm 1 \%$ for each energy in the region from $R_{100}$ (depth of dose maximum) to $R_{p}$. The disagreement was larger (a maximum of $13 \%$ ) close to the phantom surface. This difference is due to the waterproof enclosure of the parallel plane chamber, which affects the depth profile at the surface of the phantom. Table 3 lists the differences between the measured and calculated PDD values for each energy using the $10 \times 10 \mathrm{~cm}^{2}$ applicator.

The calculated OARs for each energy agreed with the measurement to within $\pm 1 \%$ inside the radiation field at both $R_{s}$ and $R_{p}$. Outside the radiation field, the calculated
OARs differed from the measurements by less than $0.5 \%$. Table 4 lists the differences between the measured and calculated OARs at $R_{\mathrm{s}}$ for each energy for the $10 \times 10 \mathrm{~cm}^{2}$ applicator.

The dose distributions measured outside the $10 \times 10 \mathrm{~cm}^{2}$ applicator at $R_{s}$ in the water phantom for the 4- and 6-MeV electron beams exhibited a local peak and a peripheral dose, as shown in Fig. 3. These peaks were located about $12 \mathrm{~cm}$ from the central beam axis for 4- and 6-MeV electron energies. The local peaks for the other energies were discrete. Because the radiation transmitted through the standard insert alloy increased as the electron energy increased, a distinct difference appeared between the dose below the standard insert alloy and that outside the applicator. The differences between the measured and calculated OAR data were less than $\pm 0.5 \%$ at both $R_{s}$ 
outside the radiation field. However, they became larger at greater distances from the central beam axis. These differences occurred because the electronic components and the lead blocks shielding the target, the monitor chamber, and the jaw inside the treatment head could not be simulated accurately and were not included in the design drawing. Because the design drawing did not include an accurate thickness for each scraper in the applicator, the error in the thickness measured using the caliper affected the results calculated outside the applicator.

\section{Fluence of scattered and transmitted radiation from the applicator}

The fluence distributions for each energy inside the radiation field at $100 \mathrm{~cm}$ SSD for the $10 \times 10 \mathrm{~cm}^{2}$ applicator are shown in Fig. 4. The shape of the distribution of the electron fluence generated by the secondary jaws and applicator inside the radiation field at $100 \mathrm{~cm}$ SSD changed very little with the electron energy. A number of electron fluence components existed on the periphery of the maximum energy. The lower-energy photon component and the photon fluence per energy bin increased as the accelerating energy increased. Figure 5 shows the peripheral fluence distributions on a plane at $100 \mathrm{~cm}$ SSD for each energy outside of the radiation field for the $10 \times 10 \mathrm{~cm}^{2}$ applicator. All of the fluence outside the applicator was generated by radiation transmitted or scattered by the secondary jaws and applicator, and a small fluence due to scattered electrons contributed to the total fluence. The fluence contributions from electrons and photons generated by the applicator exhibited similar trends for each energy. The electron fluence peripheral to the maximum energy increased at low accelerating energies. The fact that the angle of radiation scattered by the Compton effect
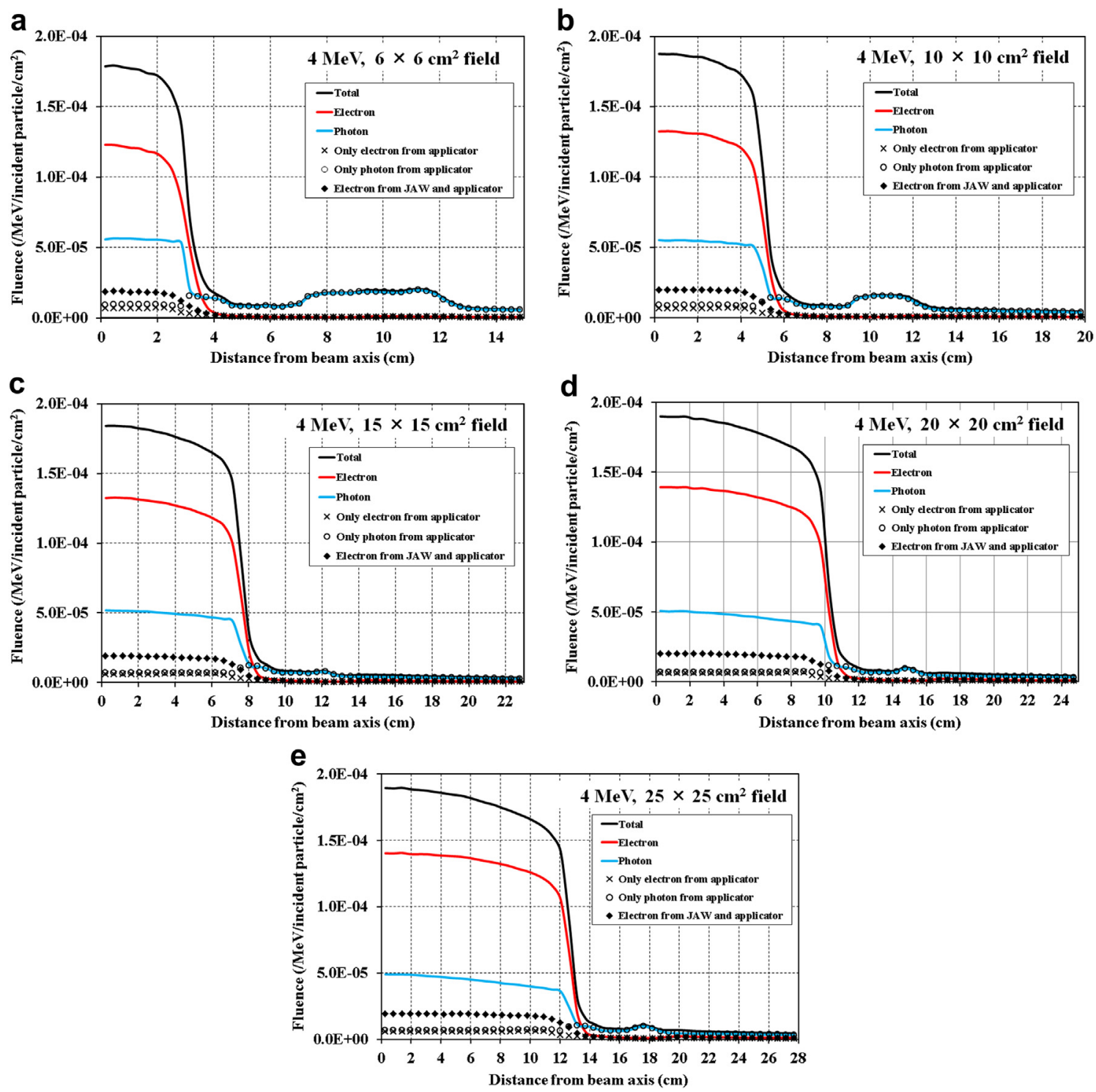

Figure 6 Fluence values in the cross-line (transverse) direction for applicator sizes of (a) $6 \times 6 \mathrm{~cm}^{2},(\mathrm{~b}) 10 \times 10 \mathrm{~cm}^{2}$, (c) $15 \times 15 \mathrm{~cm}^{2}$, (d) $20 \times 20 \mathrm{~cm}^{2}$, and (e) $25 \times 25 \mathrm{~cm}^{2}$ for the 4-MeV electron beam. 
is large for lower accelerating energies seemed to account for the increases in electron fluence outside the applicator.

Figure 6 shows the fluence distributions for various applicator sizes in the cross-line (transverse) direction for the 4-MeV electron beam at $100 \mathrm{~cm}$ SSD. The distance from the edge of the radiation field to the local peak was about $7 \mathrm{~cm}$ regardless of the applicator size. Thus, the scattering angle of radiation scattered from the applicator changed very little despite variations in the applicator size. The maximum fluence intensity at the local peak was $1.5 \%$ for the $25 \times 25 \mathrm{~cm}^{2}$ applicator. Although the photon fluence outside the applicator for the 4-MeV electron beam also showed a local peak, the dose delivered by the photon fluence did not strongly affect the dose profile in the water phantom, as shown in Fig. 3. This result shows that the contribution to the dose from secondary electrons generated by photons was low for the $4-\mathrm{MeV}$ electron beam. These results agree with the data measured by Chow and Grigorov [6] using films.
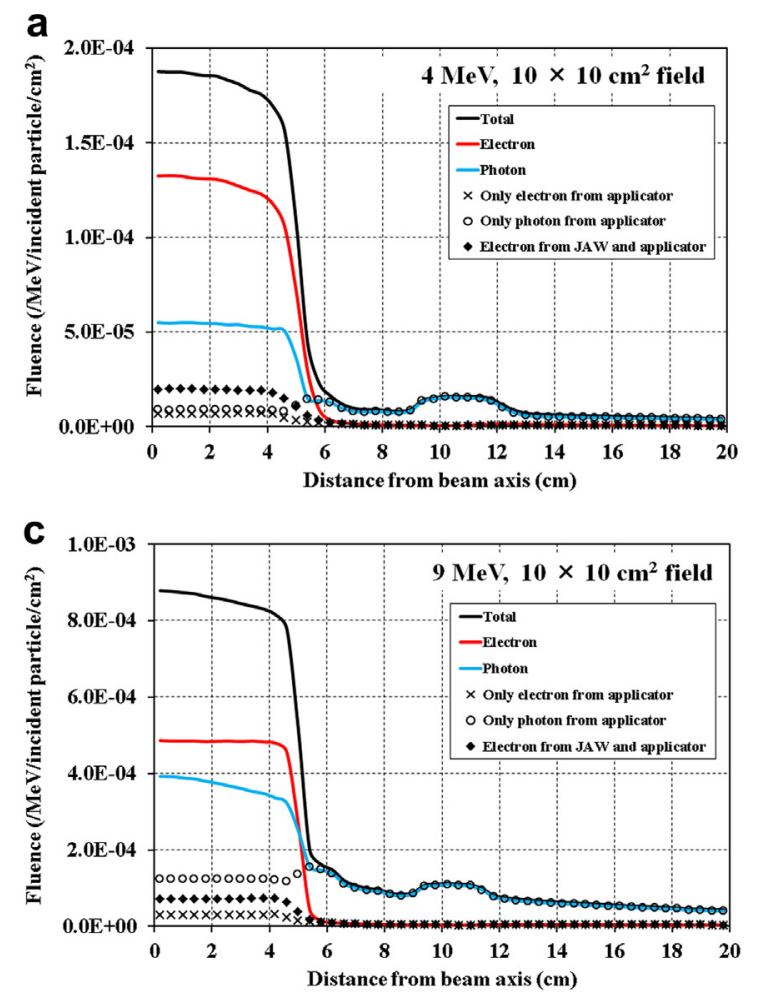

Figure 7 shows the fluence distribution for each electron energy in the cross-line direction for the $10 \times 10 \mathrm{~cm}^{2}$ applicator. At each energy, the fluence outside the applicator was produced by photons and electrons (including positrons) generated by interactions with the applicator. The fluence of photons below the "standard insert" corresponding to the field size determined at the lowest scraper increased as the accelerating energy increased. The increase in the dose between the edge of the radiation field and the local peak (Fig. 3) is attributed to enhancement by secondary electrons generated by photons impacting this region and passing through the lowest scraper.

According to our MC analysis, the fluence entered the water phantom; we investigated which components of the scattered and transmitted radiation affected the absorbed dose inside the phantom. Although the Varian-type applicator is an improvement over older applicators, its shape has many gaps and open sidewalls; however, there was very little radiation leakage, and it proved to be an excellent

b

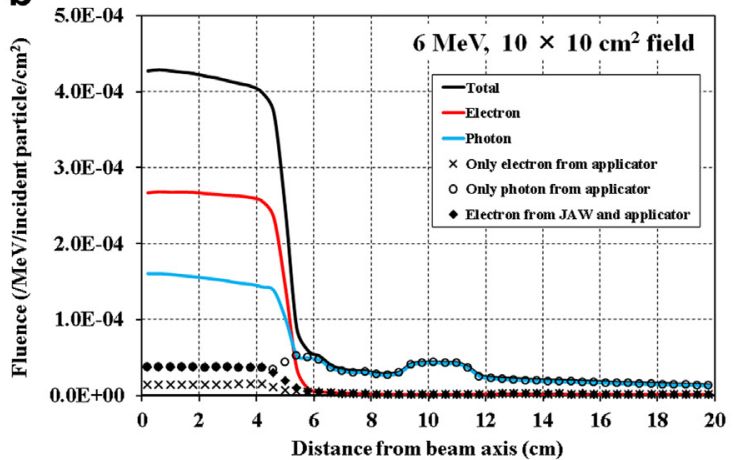

d

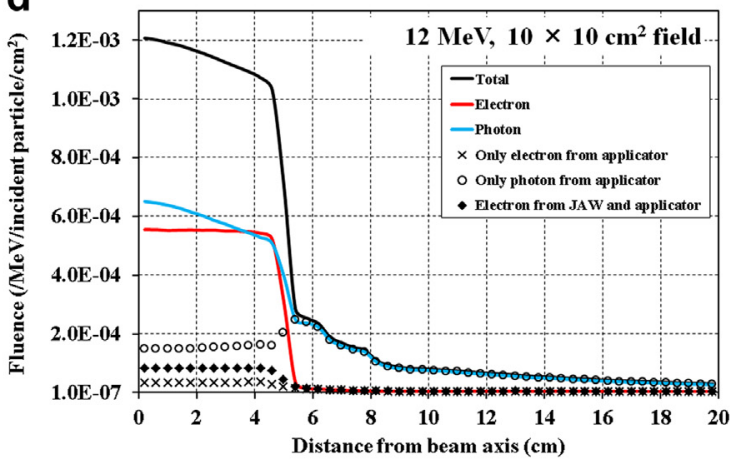

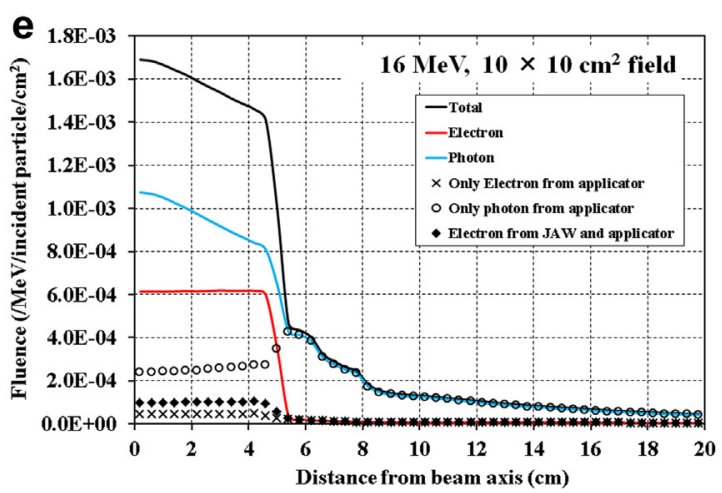

Figure 7 Fluence values in the cross-line (transverse) directions for beam energies of (a) $4 \mathrm{MeV}$, (b) $6 \mathrm{MeV}$, (c) $9 \mathrm{MeV}$, (d) $12 \mathrm{MeV}$, and (e) $16 \mathrm{MeV}$ electron beams for the $10 \times 10 \mathrm{~cm}^{2}$ applicator. 
unit. Possible methods of reducing the radiation leakage outside the applicator include increasing the scraper thickness or using a denser material for the scraper. However, these changes may reduce the flat region in the radiation field. In addition, although increasing the scraper thickness is expected to reduce the transmitted radiation below the scrapers, it may also increase the scattered radiation outside the applicator.

\section{Conclusions}

Radiation in the peripheral regions of the defined applicator fields in a Varian linac was investigated in this study. The applicator we examined was superior to the older types of applicators reported in the literature [4] and reduced the scattered radiation. The results of our study were similar to those of a previous study [6], which reported that the peripheral dose outside the applicator was about $1.5 \%$. We conducted a detailed analysis of the peripheral dose by measurements and an MC simulation. It is necessary to analyze each linear accelerator in detail because the configuration inside the accelerator head and the shape of the applicator vary depending on the type of accelerator and the manufacturer. Investigating the peripheral dose is prudent because it is usually not fully accounted for by commercial treatment planning systems.

This study analyzed only the square fields formed by the alloy used for the standard insert during beam data measurement and not the complex fields formed by low-melting lead alloy. Such research will be conducted in the future.

\section{References}

[1] Khan FM. The physics of radiation therapy. 3rd ed. Philadelphia, PA: Lippincott Williams \& Wilkins; 2003. p. 340-8.

[2] Richard A, Keys MA, Purdy JA. Radiation leakage from linac electron applicator assembly. Int J Radiat Oncol Biol Phys 1984;10(5):713-21.

[3] Das KR, Cramb JA, Millar M, Kenny MB, Patterson WR, Ackerly TL, et al. Levels of leakage radiation from electron collimators of a linear accelerator. Med Phys 1990;17(6): 1058-63.

[4] Perec A, Kubo H. Radiation leakage through electron applicators on clinac-1800 accelerators. Med Phys 1990;17(4):715-9.

[5] Pennington EC, Jani SK, Wen BC. Leakage radiation from electron applicators on a medical accelerator. Med Phys 1988; 15(5):763-5.

[6] Chow JCL, Grigorov GN. Peripheral dose outside applicators in electron beams. Phys Med Biol 2006;51:N231-40.

[7] Wen BC, Pennington EC, Hussey DH, Jani SK. Alopecia associated with unexpected leakage from electron cone. Int J Radiat Oncol Biol Phys 1989;16(6):1637-41.
[8] Kassaee A, Altschuler MD, Ayyalsomayajula S, Bloch P. Influence of cone design on the electron beam characteristics on clinical accelerators. Med Phys 1994;21(11):1671-6.

[9] Yeboah C, Karotki A, Hunt D, Holly R. Quantification and reduction of peripheral dose from leakage radiation on siemens primus accelerators in electron therapy mode. J Appl Clin Med Phys 2010;11(3):154-72.

[10] Edimo P, Clermont C, Kwato MG, Vynckier S. Evaluation of a commercial $\mathrm{VMC}++$ Monte Carlo based treatment planning system for electron beams using EGSnrc/BEAMnrc simulations and measurements. Phys Med 2009; 25:111-21.

[11] O’Shea TP, Sawkey DL, Foley MJ, Faddegon BA. Monte Carlo commissioning of clinical electron beams using large field measurements. Phys Med Biol 2010;55:4083-105.

[12] Battum LJ, Zee W, Huizenga $H$. Scattered radiation from applicators in clinical electron beams. Phys Med Biol 2003;48: 2493-507.

[13] Zhang GG, Rogers DWO, Cygler JE, Mackie TR. Monte Carlo investigation of electron beam output factors versus size of square cutout. Med Phys 1999;26(5):743-50.

[14] Ebert MA, Hoban PW. A model for electron-beam applicator scatter. Med Phys 1995;22(9):1419-29.

[15] Ebert MA, Hoban PW. A Monte Carlo investigation of electronbeam applicator scatter. Med Phys 1995;22(9):1431-5.

[16] Monte Carlo data package. Varian medical systems. DWO No.100040466-100040501 rev.1.

[17] Almond PR, Biggs PJ, Coursey BM, Hanson WF, Huq MS, Nath R, et al. AAPM's TG-51 protocol for clinical reference dosimetry of high-energy photon and electron beams. Med Phys 1999; 26(9):1847-70.

[18] Khan FM, Doppke KP, Hogstrom KR, Kutcher GJ, Nath R, Prasad SC, et al. Clinical electron-beam dosimetry: report of AAPM radiation therapy committee task group no. 25. Med Phys 1991;18(1):73-109.

[19] Kawrakow I, Mainegra-Hing E, Rogers DWO, Tessier F, Walters BRB. The EGSnrc code system: Monte Carlo simulation of electron and photon transport. NRCC report PIRS-701; 2011.

[20] Rogers DWO, Faddegon BA, Ding GX, Ma CM, We J, Mackie TR. BEAM: a Monte Carlo code to simulate radiotherapy treatment units. Med Phys 1995;22(5):503-24.

[21] Rogers DWO, Walters B, Kawrakow I. BEAMnrc users manual. NRCC report PIRS-0509(A)revL; 2011.

[22] Walters B, Kawrakow I, Rogers DWO. DOSXYZnrc users manual. NRCC report PIRS-794revB; 2011.

[23] Kawrakow I, Mainegra-Hing E, Rogers DWO. EGSnrcMP: the multi-platform environment for EGSnrc. NRCC report PIRS877; 2006.

[24] MCGowan HCE, Faddegon BA, Ma CM. STATDOSE for 3D dose distributions. NRCC report PIRS-0509(F); 2007.

[25] Kutcher GJ, Coia L, Gillin M, Leibel S, Morton RJ, Palta JR, et al. Comprehensive QA for radiation oncology: report of AAPM radiation therapy committee task group 40 . Med Phys 1994;21(4):581-618.

[26] Ma CM, Rogers DWO. BEAMDP users manual. NRCC report PIRS0509(C)revA; 2009. 